\title{
Brain-Targeted Proanthocyanidin Metabolites for Alzheimer's Disease Treatment
}

\author{
Jun Wang, ${ }^{1,9}$ Mario G. Ferruzzi, ${ }^{3}$ Lap Ho, ${ }^{1,9}$ Jack Blount, ${ }^{5}$ Elsa M. Janle, ${ }^{3}$ Bing Gong, ${ }^{1}$ Yong Pan, ${ }^{1}$ G. A. Nagana Gowda, \\ Daniel Raftery, ${ }^{4}$ Isabel Arrieta-Cruz, ${ }^{1}$ Vaishali Sharma, ${ }^{5}$ Bruce Cooper, ${ }^{3}$ Jessica Lobo, ${ }^{3}$ James E. Simon, ${ }^{7}$ \\ Chungfen Zhang, ${ }^{5}$ Alice Cheng, ${ }^{1}$ Xianjuan Qian, ${ }^{1}$ Kenjiro Ono, ${ }^{8}$ David B. Teplow, ${ }^{8}$ Constantine Pavlides, ${ }^{6}$ \\ Richard A. Dixon, ${ }^{5}$ and Giulio M. Pasinetti ${ }^{1,2,9}$ \\ ${ }^{1}$ Department of Neurology and ${ }^{2}$ Department of Psychiatry, Mount Sinai School of Medicine, New York, New York 10029, ${ }^{3}$ Departments of Food Science and \\ Foods and Nutrition and ${ }^{4}$ Department of Chemistry, Purdue University, West Lafayette, Indiana 47907, ${ }^{5}$ Plant Biology Division, Samuel Roberts Noble \\ Foundation, Ardmore, Oklahoma 73401, ${ }^{6}$ The Rockefeller University, New York, New York 10021, ${ }^{7}$ Department of Plant Biology and Plant Pathology, \\ Rutgers, the State University of New Jersey, New Brunswick, New Jersey 08901, ${ }^{8}$ Department of Neurology, David Geffen School of Medicine, and Brain \\ Research Institute, and Molecular Biology Institute, University of California, Los Angeles, California 90095, and 99Geriatric Research, Education and Clinical \\ Center, James J. Peters Veterans Affairs Medical Center, Bronx, New York 10468
}

While polyphenolic compounds have many health benefits, the potential development of polyphenols for the prevention/treatment of neurological disorders is largely hindered by their complexity as well as by limited knowledge regarding their bioavailability, metabolism, and bioactivity, especially in the brain. We recently demonstrated that dietary supplementation with a specific grape-derived polyphenolic preparation (GP) significantly improves cognitive function in a mouse model of Alzheimer's disease (AD). GP is comprised of the proanthocyanidin (PAC) catechin and epicatechin in monomeric (Mo), oligomeric, and polymeric forms. In this study, we report that following oral administration of the independent GP forms, only Mo is able to improve cognitive function and only Mo metabolites can selectively reach and accumulate in the brain at a concentration of $\sim 400 \mathrm{~nm}$. Most importantly, we report for the first time that a biosynthetic epicatechin metabolite, $3^{\prime}$ - 0 -methyl-epicatechin-5-O- $\beta$-glucuronide (3'-O-Me-EC-Gluc), one of the PAC metabolites identified in the brain following Mo treatment, promotes basal synaptic transmission and long-term potentiation at physiologically relevant concentrations in hippocampus slices through mechanisms associated with cAMP response element binding protein (CREB) signaling. Our studies suggest that select brain-targeted PAC metabolites benefit cognition by improving synaptic plasticity in the brain, and provide impetus to develop 3'-O-Me-EC-Gluc and other brain-targeted PAC metabolites to promote learning and memory in AD and other forms of dementia.

\section{Introduction}

Plant-derived polyphenolic compounds possess diverse biological activities, including strong antioxidant, anti-inflammatory, antimicrobial, and antitumorogenic activities. There is a growing interest in the development of polyphenolic compounds for preventing or treating chronic and degenerative diseases such as cardiovascular disorders, cancer, as well as neurological diseases including Alzheimer's disease (AD).

Our laboratory and others have shown that polyphenolic compounds from multiple dietary sources, including a specific

\footnotetext{
Received Dec. 23, 2011; revised Feb. 24, 2012; accepted Feb. $27,2012$.

Author contributions: J.W., M.G.F., L.H., E.M.J., D.R., J.E.S., D.B.T., C.P., R.A.D., and G.M.P. designed research; J.W., J.B., E.M.J., B.G., Y.P., G.A.N.G., I.A.-C., V.S., B.C., J.L., C.Z., A.C., X.Q., and K.O. performed research; J.W., M.G.F., E.M.J., B.G., G.A.N.G., D.R., J.E.S., D.B.T., C.P., R.A.D., and G.M.P. analyzed data; J.W., M.G.F., L.H., and G.M.P. wrote the paper.

This work was supported by grants from the NIH (P01AT004511) and Department of Veterans Affairs to G.M.P., the Jim Easton Consortium for Alzheimer's Drug Discovery and Biomarkers at University of California Los Angeles to D.B.T., NIH Grant AG027818 to D.B.T., and the Samuel Roberts Noble Foundation (R.A.D.).

Correspondence should be addressed to Dr. Giulio M. Pasinetti, Department of Neurology, The Mount Sinai School of Medicine, 1 Gustave L. Levy Place, Box 1137, New York, NY 10029. E-mail: giulio.pasinetti@mssm.edu.

DOI:10.1523/JNEUROSCI.6437-11.2012

Copyright $\odot 2012$ the authors $\quad 0270-6474 / 12 / 325144-07 \$ 15.00 / 0$
}

grape-derived polyphenolic preparation (GP), are capable of attenuating cognitive deterioration and reducing brain neuropathology in animal models of AD (Rezai-Zadeh et al., 2005; Hartman et al., 2006; Wang et al., 2008). GP is a complex mixture of proanthocyanadins (PACs) consisting of flavan-3-ol units including catechin (C), epicatechin (EC), catechin gallate, and epicatechin gallate. These monomeric units form the basis of various types of oligomers and polymers through $\mathrm{C} 4 \rightarrow \mathrm{C} 8$ or $\mathrm{C} 4 \rightarrow \mathrm{C} 6$ interflavan bonds (Sharma et al., 2011). PACs are the most abundant and complex polyphenols in grapes and grape-derived products.

The application of phytotherapeutic agents, including GP and other PAC-rich natural compounds, in the treatment of neurological disorders is largely hindered by the limited knowledge of their metabolisms, bioactivities, and whether sufficient concentrations can reach and accumulate in the brain to exert biological activities.

Our studies were designed to explore the pharmacokinetics (PK) of specific GP components and the mechanistic basis of their bioactivities in the brain. Our studies also tested whether a biosynthetic brain-targeted PAC metabolite could recapitulate 
the biological activity of GP, and provide insight on the development of "phytodrugs" as novel therapeutic agents for the attenuation of cognitive deterioration in $\mathrm{AD}$ and other forms of dementia.

\section{Materials and Methods}

Chemicals and materials. (+)-Catechin (C), (-)-epicatechin (EC), and gallic acid standards were purchased from Sigma-Aldrich. 3'-O-methylepicatechin $\left(3^{\prime}-O-M e-E C\right)$ was from Nacalai USA. All extraction and liquid chromatography (LC) solvents were of certified HPLC and American Chemical Society grade from J.T. Baker. GP was obtained from Polyphenolics.

Fractionation of GP. GP were extracted in acetone:water (7:3) under $\mathrm{N}_{2}$ and dissolved in Milli-Q water (Millipore) and re-extracted three times with equal volumes of ethyl acetate. The organic phase was evaporated, redissolved, and applied to an solid phase extraction (SPE) Column (ENV1 18, 10 g; Supelco). The PAC monomer (Mo)-enriched fraction was eluted with diethyl ether, evaporated, redissolved in water, and freezedried. The aqueous layer containing PAC oligomers and polymers (Po) was evaporated, freeze-dried, and fractionated as described previously (Sharma et al., 2011).

$A D$ mice and treatment. Female Tg2576 AD transgenic mice were purchased from Taconic and all procedures were approved by the Mount Sinai School of Medicine Institutional Animal Care and Use Committee. Mice were randomized into the nontreated control group, the Motreated group, or the Po-treated group. Animals were treated for 5 months starting at 7 months of age before the development of AD neuropathology/cognitive deficits. Both Mo and Po were delivered through their drinking water (Wang et al., 2008). The Mo-treated group was treated with $80 \mathrm{mg} / \mathrm{kg} / \mathrm{d} \mathrm{Mo}$, equivalent to the amount of monomer PACs in $200 \mathrm{mg} / \mathrm{kg} / \mathrm{d} \mathrm{GP}$, which was used in our previous study (Wang et al., 2008). The Po-treated group was treated with $120 \mathrm{mg} / \mathrm{kg} / \mathrm{d}$ Po, equivalent to the amount of polymer PACs from $200 \mathrm{mg} / \mathrm{kg} / \mathrm{d}$ GP.

Behavioral assessment by the Morris water maze test. Spatial learning memory was assessed by the Morris water maze behavioral test when the mice were $\sim 12$ months of age following 5 months treatment, as previously described (Wang et al., 2007).

Assessment of AD-type amyloid neuropathology. Total $\mathrm{A} \beta_{1-40}$ or $\mathrm{A} \beta_{1-42}$ in the brain were quantified by sandwich ELISA (BioSource), as previously described (Wang et al., 2005). The level of soluble A $\beta$ oligomers was measured by a commercially available sandwich ELISA (Wang et al., 2008) according to the manufacturer's instruction. Specifically, soluble amyloid peptide was extracted in PBS and centrifuged at 78,500 $\times \mathrm{g}$ for $1 \mathrm{~h}$ at $4^{\circ} \mathrm{C}$, and the supernatant was quantified by ELISA to specifically detect aggregated $\mathrm{A} \beta$ (Invitrogen).

Bioavailability, metabolism, and brain penetration of GP and fractions. Eight-week-old male Sprague Dawley (SD) rats were placed on a polyphenol-free AIN-93M diet. Doses of Mo and Po were designed to match monomer and polymer PAC doses from GP, respectively, which are $41 \mathrm{mg} / \mathrm{kg}$ body weight (BW) GP, $17 \mathrm{mg} / \mathrm{kg}$ BW Mo, and $28.3 \mathrm{mg} / \mathrm{kg}$ BW Po. Doses of GP and fractions were administered to rats over a $10 \mathrm{~d}$ period through gavage. On day 11, animals were administered a final dose and killed an hour later by compressed $\mathrm{CO}_{2}$. The brain was harvested following PBS perfusion and placed in saline $(0.2 \%$ ascorbic acid) and stored at $-80^{\circ} \mathrm{C}$ until analysis.

Extraction and analysis of $C$ metabolites from brain tissues. $\mathrm{C}$ and $\mathrm{EC}$ metabolites were extracted from brain tissues by solid phase extraction. Briefly, $\sim 500 \mathrm{mg}$ of brain tissues were extracted with methanol, dried, and resolubilized in $1.5 \mathrm{~m}$ formic acid before loading onto preactivated Oasis HLB SPE cartridges (Waters). The C metabolites were eluted with acidified methanol, vacuum-dried, sonicated, and resolubilized in mobile phase before LC-MS analysis.

Analysis of Cs from the brain was performed using an Agilent 6400 Series QQQ in multiple reaction monitoring (MRM) mode using identical ionization conditions used on the time-of-flight (TOF) with $30 \mathrm{eV}$ collision energy used for MS/MS experiments. C and EC metabolite quantifications were estimated using calibration curves from parent standard compounds.
Electrophysiological recordings. Tg2576 mice aged 22-24 months were used to assess the effect of 3 '-O-Me-EC- $\beta$-Gluc on basal synaptic transmission and long-term potentiation (LTP). This model of AD is known to exhibit significant synaptic impairments at this age (Chapman et al., 1999). Hippocampal slices $(350 \mu \mathrm{m})$ were acclimated in oxygenated artificial CSF and treated with $300 \mathrm{~nm} 3^{\prime}-O-M e-E C-\beta$-Gluc for $4-5 \mathrm{~h}$. The field EPSPs (fEPSPs) were recorded from the CA1 region as described previously (Gong et al., 2004). LTP was induced using theta-burst stimulation (four pulses at $100 \mathrm{~Hz}$, with the bursts repeated at five $\mathrm{Hz}$, and each tetanus including three 10-burst trains separated by $15 \mathrm{~s}$ ).

Multipathway cell signaling assays and Western blot analysis. Luminex xMAP multiplexed immunoassays (Millipore) were used to evaluate the levels of phosphorylated proteins: CREB (Ser133), Erk/MAP kinase 1/2 (Thr185/Tyr187), AKT (Ser473), JNK (Thr183/Tyr185), p70 S6 kinase (Thr412), MEK1 (Ser222), p38 (Thr189/Tyr182), and Rsk1 (Ser380) in

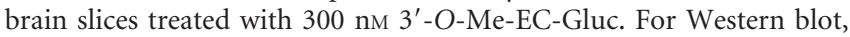
samples were separated by SDS-PAGE and antibodies specific for phosphor-CREB, total CREB (Millipore), phosphor-CaMKII, total CaMKII, and $\beta$-tubulin (Santa Cruz Biotechnology) were used as probes.

Statistical analysis. Data were analyzed using Prism software (V4.03; GraphPad Software). Data are presented as mean \pm SEM and analyzed using two-way ANOVA with repeated measure (RM), one-way ANOVA followed by Bonferroni's post hoc tests, or two-tailed Student's $t$ test. In all analyses, the null hypothesis was rejected at the 0.05 level.

\section{Results}

\section{Fractionation of GP}

Based on reverse-phase HPLC analysis with detection by MS, or normal-phase HPLC analysis with detection by postcolumn derivatization, unfractionated intact GP is comprised of increasing proportions of monomeric, oligomeric, and polymeric proanthocyanidin components (Fig. $1 A$ ). In comparison, the Mo preparation is highly enriched in the monomeric components (Fig. $1 B$ ), whereas the Po preparation is comprised of predominantly polymeric components with traces $(<1 \%)$ of Mo components (Fig. 1C).

In an in vitro analysis using the photo-induced cross-linking of unmodified proteins (PICUP) technique, we found that, similar to GP, both Mo and Po are capable of interfering with the initial protein-protein interaction of $\mathrm{A} \beta_{1-40}$ and $\mathrm{A} \beta_{1-42}$ that is necessary for the formation of neurotoxic oligomeric $A \beta$ species (data not shown).

\section{Dietary supplementation with Mo, not Po, improves cognitive function in $\mathrm{AD}$ mice}

To explore the potential roles of specific components of GP in protection against $\mathrm{AD}$-type cognitive deterioration, we treated Tg2576 mice with either Mo or Po. The Morris water maze (MWM) behavior test showed that Mo treatment significantly improved the cognitive behavioral performance of Tg2576 mice following 5 months of treatment, as reflected by a significant time-dependent decrease in the latency for finding the submerged escape platform compared with nontreated control Tg2576 mice ( $p<0.01$, two-way RM-ANOVA; Fig. $1 D)$. In contrast, Po treatment did not lead to detectable improvements (Fig. $1 D)$. In the probe trial phase of the MWM, Mo-treated Tg2576 mice, compared with Po-treated mice and nontreated control mice, spent significantly more time in the target quadrant area compared with the other quadrants (one-way ANOVA, $p<$ 0.005; Fig. $1 E$ ), confirming that Mo treatment significantly improved spatial memory retention. The treatment did not affect nonspatial parameters such as swimming speed (Fig. $1 F$ ) that might interfere with MWM test.

In parallel control studies to test whether the cognitive improvement is associated with $\mathrm{A} \beta$-mediated mechanism, we 
A

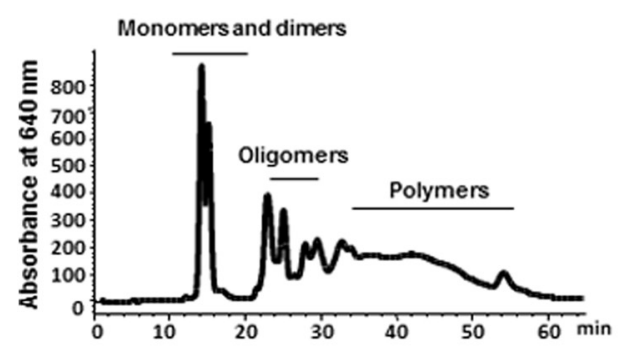

B

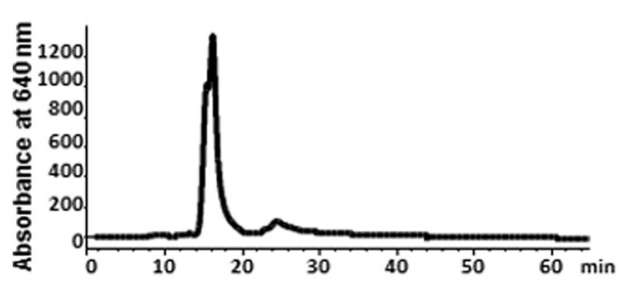

C

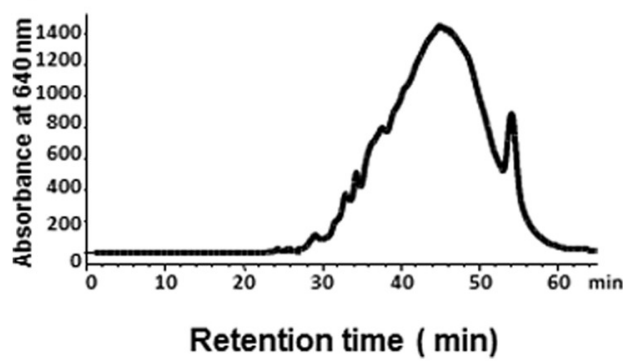

D

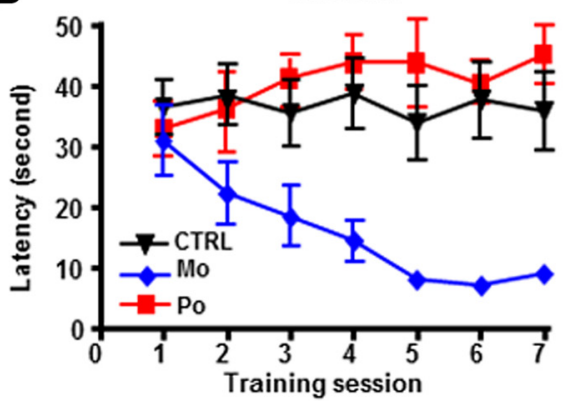

E

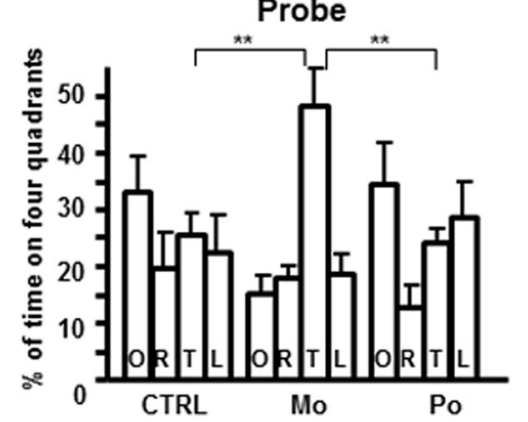

F

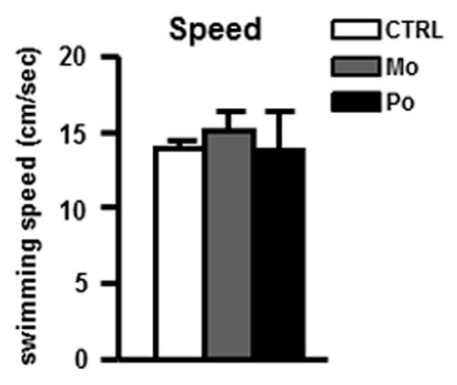

G

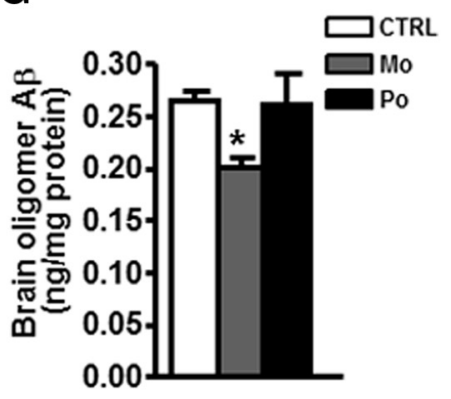

H

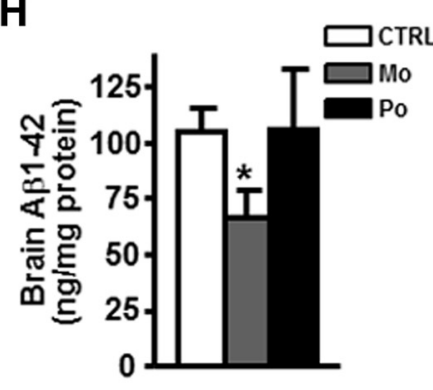

I

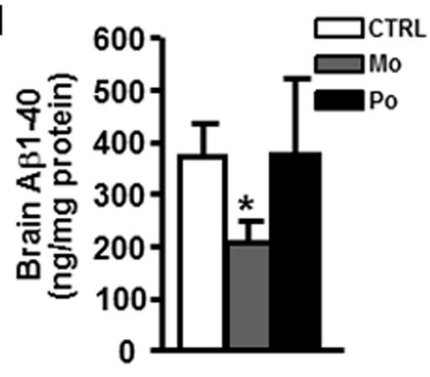

Figure 1. Fractionation of GP and in vivo efficacy of Mo and Po on A $\beta$-related neuropathology in Tg2576 mice. $\boldsymbol{A}-\boldsymbol{C}$, Normal phase HPLC chromatograms of GP fractions: GP (A), Mo-enriched fraction $(\boldsymbol{B})$, Po-enriched fraction $(\boldsymbol{C})$. D-F, The influence of chronic Mo or Po treatment on A $\beta$-related spatial memory in Tg2576 mice using MWM test. $\boldsymbol{D}$, Hidden platform acquisition; latency score represents the time taken to escape to the platform. $\boldsymbol{E}$, Probe trial. Percentage of time in four different quadrants (T, target; 0 , opposite; R, right; $L$, left). $\boldsymbol{F}$, Swimming speed. $\mathbf{G}-\mathbf{I}, \mathbf{Q}$ uantifications of oligomeric $A \beta(\boldsymbol{G})$, total $A \beta_{1-42}(\boldsymbol{H})$, and $A \beta_{1-40}(\boldsymbol{I})$ in brains of Mo-treated, P0-treated, or control (CTRL) mice using ELISA assay. Data represents mean \pm SEM, $n=8-10$ mice per group. ${ }^{*} p<$ $0.05,{ }^{* *} p<0.01$.

found that similar Mo treatment did not alter MWM performance in strain- and gender-matched wild-type mice (data not shown).

Neuropathology analysis also showed that compared with nontreated control Tg2576 mice, Mo treatment significantly reduced the content of oligomeric $\mathrm{A} \beta$ species (Fig. $1 G$ ) as well as the contents of $\mathrm{A} \beta_{1-42}$ (Fig. $1 H$ ) and $\mathrm{A} \beta_{1-42}$ (Fig. $1 I$ ) in the brain while no detectable changes were observed following Po treatments.

Plasma pharmacokinetic response for $\mathrm{C}$ and $\mathrm{EC}$ metabolites from GP and specified fractions

A major consideration for the potential development of polyphenolics for treating neurodegenerative disorders is bioavailability, particularly in the brain. In this study, we used SD rats. The choice of SD rats was based on their well established use as a model for bioavailability and metabolism of polyphenols in humans and based on the fact that both rats and mice possess similar xenobiotic enzyme systems, and similar metabolites of $\mathrm{C}$ and EC, namely methylated and glucuronidated metabolites, are observed in both species in previous studies (Feng, 2006).

Polyphenol PK studies are traditionally conducted using a single, acute dose paradigm. However, a repeated dose paradigm more accurately reflects polyphenol $\mathrm{PK}$ in response to long-term application of polyphenols for clinical application. We found that metabolites of PAC monomers, specifically $\mathrm{C}$ and EC, were detected in rat plasma following $10 \mathrm{~d}$ of GP or Mo treatment (Fig. $2 A)$. The predominant plasma metabolites of $\mathrm{C}$ and $\mathrm{EC}$ were identified by LC-MS-TOF as (-)-C-O- $\beta$-glucuronide, $3^{\prime}-O$ methyl-( \pm )-C-O- $\beta$-glucuronide, ( - -EC- $O$ - $\beta$-glucuronide, and $3^{\prime}$-O-Me-EC-Gluc (Fig. $2 \mathrm{~A}$, from left to right). Characterization of these metabolites is consistent with previous reports (Roura et al., 2005; Tsang et al., 2005). Only small levels of the same C and EC methylated and glucuronidated metabolites were found in the plasma following Po treatment (Fig. $2 \mathrm{~A}$ ). There were no quantifiable levels of PAC dimers, trimers, or larger oligomeric PACs in the plasma following oral administration of GP, Mo, or Po.

Compared with single acute dosage, bioavailability of $\mathrm{C}$ and EC metabolites was significantly higher following repeated GP or Mo dosing, as summarized in Table 1. Both area under the curve (AUC) and $C_{\max }$ values were significantly higher $(p<0.01)$ for each of the $\mathrm{C}$ and $\mathrm{EC}$ metabolites following repeated dosing compared with single acute treatment with identical doses of intact GP or Mo (Table 1). AUC values for total C and EC glucuronides increased by $\sim 10$ - to 20 -fold, with the largest increases observed 

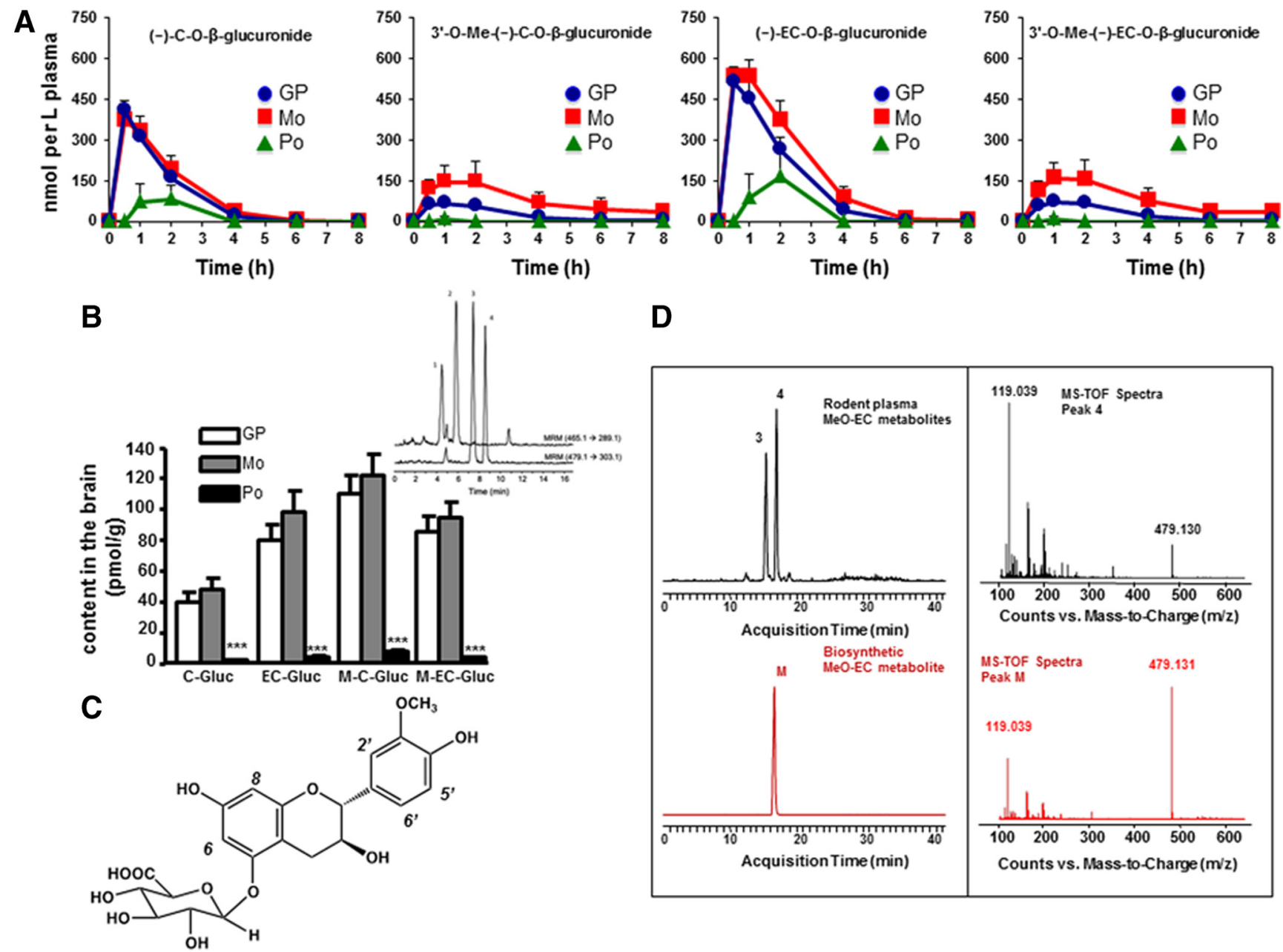

D

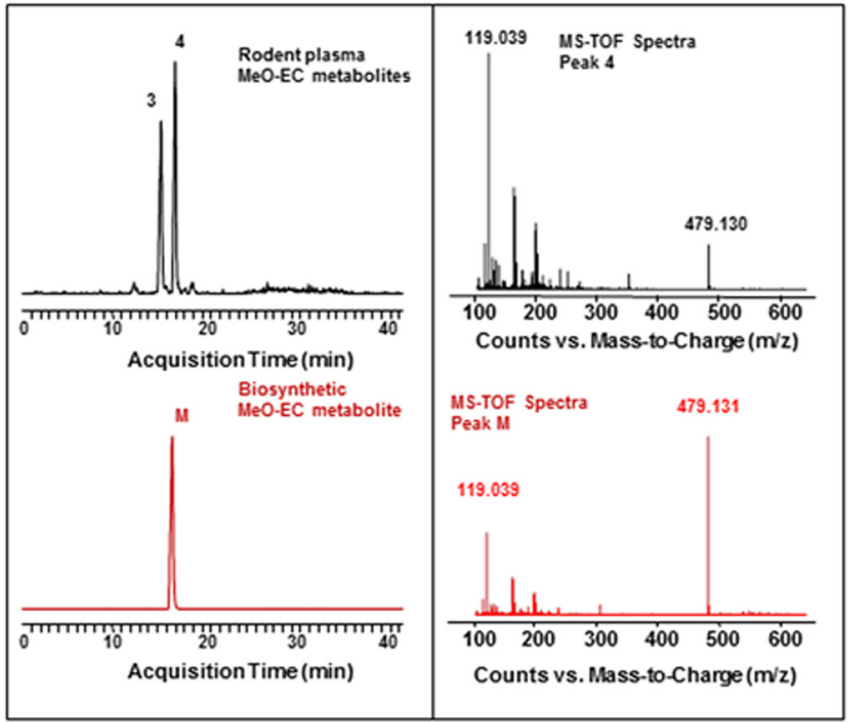

Figure 2. Plasma pharmacokinetics, brain levels of $\mathrm{C}$ and EC metabolites, and structural characterization of biosynthetic EC metabolite. $A$, Plasma pharmacokinetic profile of major $\mathrm{C}$ and EC metabolites following repeated dosing of rats by treatment with GP, Mo, and Po. B, Concentration of C and EC metabolites in brain tissue following $10 \mathrm{~d}$ of treatment. Inset, LC-MS/MS separation of major $\mathrm{C}$ and $\mathrm{EC}$ metabolites detected in extracts of rat brain tissue collected after $10 \mathrm{~d}$ of treatment. MRM trace is shown for $\mathrm{C} / \mathrm{EC}-0-\beta-$ glucuronide $(465.1 \rightarrow 289.1 \mathrm{~m} / \mathrm{z})$ and MeO-C/EC- $0-\beta$ glucuronide $(479.1 \rightarrow 303.1 \mathrm{~m} / \mathrm{z})$. Peak identifications: peak 1: ( \pm )-C-0- $\beta$-glucuronide; peak 2: (-)-EC-0- $\beta$-glucuronide; peak 3: $3^{\prime}-0-M e-( \pm)-\left(-0-\beta\right.$-glucuronide; peak 4: $3^{\prime}-0-M e-(-)-E C-0-\beta$ glucuronide. ${ }^{* * *} p<0.001, n=5$ per group. C, Proposed structure of the primary EC metabolite identified as 3 '-0-Me-(-)-EC-5-0- $\beta$-glucuronide present in blood and brain tissues following repeated dosing of rats by treatment with GP or Mo fraction. D, LC-MS-TOF separation and online spectra of C and EC metabolites detected in extracts of rat plasma (black) and biosynthetic EC metabolite (red). Extracted ion chromatogram is shown for Me0-C/EC-0- $\beta$-glucuronide (479.13). Peak identifications: peak 3: 3' -0 -Me-( \pm )- $\left(-5-0-\beta\right.$-glucuronide; peak 4: $3^{\prime}-0-M e-(-)-E C-5-0-$ $\beta$-glucuronide; peak M: 3'-0-Me-(-)-EC-5-0- $\beta$-glucuronide.

Table 1. Eight-hour pharmacokinetic parameters from male Sprague Dawley rats $(n=5)$ treated with a single dose of GP (41 mg/kg BW), Mo (17 mg/kg BW), or Po (28.3 $\mathrm{mg} / \mathrm{kg} \mathrm{BW}$ ) or after repeated exposure to extracts for $10 \mathrm{~d}$

\begin{tabular}{|c|c|c|c|c|c|c|c|}
\hline \multirow[b]{2}{*}{ Metabolite } & \multirow[b]{2}{*}{ Treatment } & \multicolumn{3}{|l|}{ Single acute dose } & \multicolumn{3}{|c|}{ Acute dose following $10 \mathrm{~d}$ repeated exposure } \\
\hline & & $\begin{array}{l}\operatorname{AUC}_{(0-8 \mathrm{~h})} \\
\left(\mathrm{nmol} / \mathrm{L}^{*} \mathrm{~h}\right)\end{array}$ & $C_{\max }(\mathrm{nmol} / \mathrm{L})$ & $\mathrm{T}_{1 / 2}(\mathrm{~h})$ & $\begin{array}{l}\operatorname{AUC}_{(0-8 \mathrm{~h})} \\
\left(\mathrm{nmol} / \mathrm{L}^{*} \mathrm{~h}\right)\end{array}$ & $C_{\max }(\mathrm{nmol} / \mathrm{L})$ & $\mathrm{T}_{1 / 2}(\mathrm{~h})$ \\
\hline \multirow[t]{3}{*}{$(-)-(-0-\beta$-glucuronide } & GP & $142.2 \pm 33.9^{a}$ & $65.8 \pm 13.4^{a}$ & $1.5 \pm 0.4^{a}$ & $711.8 \pm 35.2^{* a}$ & $407.8 \pm 35.4^{* a}$ & $0.9 \pm 0.2^{a}$ \\
\hline & Mo & $151.2 \pm 24.1^{a}$ & $70.8 \pm 9.3^{a}$ & $1.3 \pm 0.2^{a}$ & $807.4 \pm 131.4^{* a}$ & $394.6 \pm 48.7^{* a}$ & $0.9 \pm 0.1^{a}$ \\
\hline & Po & ND & ND & & $179.3 \pm 73.9^{b}$ & $154.5 \pm 67.9^{b}$ & ND \\
\hline \multirow[t]{2}{*}{ (-)-EC-0- $\beta$-glucuronide } & GP & $223.9 \pm 58.2^{a}$ & $91.0 \pm 17.5^{a}$ & $1.3 \pm 0.1^{a}$ & $1063.4 \pm 98.3^{* a}$ & $532.9 \pm 29.2^{* a}$ & $1.1 \pm 0.4^{a}$ \\
\hline & Mo & $282.2 \pm 31.2^{a}$ & $110.8 \pm 14.4^{a}$ & $1.6 \pm 0.3^{a}$ & $1445.1 \pm 237.4^{* a}$ & $586.6 \pm 78.8^{* a}$ & $0.9 \pm 0.2^{a}$ \\
\hline \multirow{2}{*}{$3^{\prime}-0-$ Me-(-)-C-0- $\beta$-glucuronide } & Mo & $183.2 \pm 25.3^{a}$ & $42.8 \pm 5.1^{a}$ & $3.1 \pm 0.3^{b}$ & $938.5 \pm 598.7^{* b}$ & $177.4 \pm 70.4^{* b}$ & $2.4 \pm 0.7^{b}$ \\
\hline & Po & ND & ND & ND & $6.5 \pm 6.5^{c}$ & $8.7 \pm 8.7^{c}$ & ND \\
\hline \multirow{3}{*}{$3^{\prime}$-0-Me-(-)-EC-0- $\beta$-glucuronide } & GP & $187.7 \pm 42.5^{a}$ & $51.9 \pm 5.9^{a}$ & $2.0 \pm 0.2^{a}$ & $241.7 \pm 64.6^{* a}$ & $72.4 \pm 16.8^{* a}$ & $1.4 \pm 0.3^{a}$ \\
\hline & Mo & $216.0 \pm 32.2^{a}$ & $46.7 \pm 4.4^{a}$ & $2.8 \pm 0.2^{b}$ & $952.0 \pm 513.9^{* b}$ & $184.2 \pm 66.3^{* b}$ & $2.4 \pm 0.3^{b}$ \\
\hline & Po & ND & ND & ND & $7.3 \pm 7.3^{c}$ & $9.7 \pm 9.7^{c}$ & ND \\
\hline
\end{tabular}

Doses of GP, Mo and Po were administered as a single intragastric gavage as described in the Materials and Methods section. $A U C_{(0-8)}$, Plasma area under the curve; $C_{\max }$, maximum plasma concentration; $\mathrm{T}_{1 / 2}$, elimination half-life; $N D_{\text {, }}$ not determined.

$a, b, c$ Significant difference between single-dose treatments $(p<0.05)$. *Significant difference $(p<0.01)$ between single acute dose and repeated dose parameters. 
A
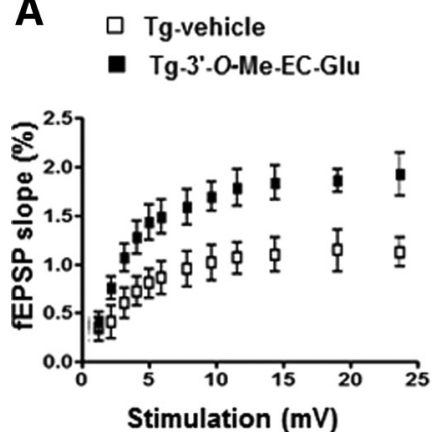

E

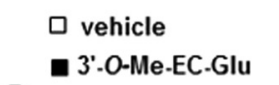

$F$
B

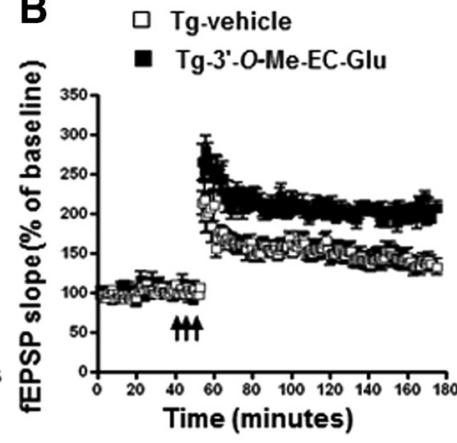

G

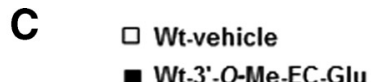

- Wt-3'-O-Me-EC-Glu

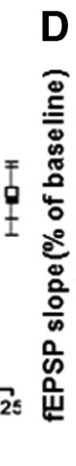

D Wt-vehicle

- Wt-3'-O-Me-EC-Glu

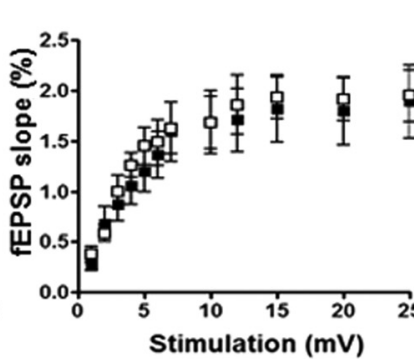

H

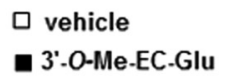

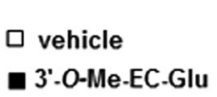
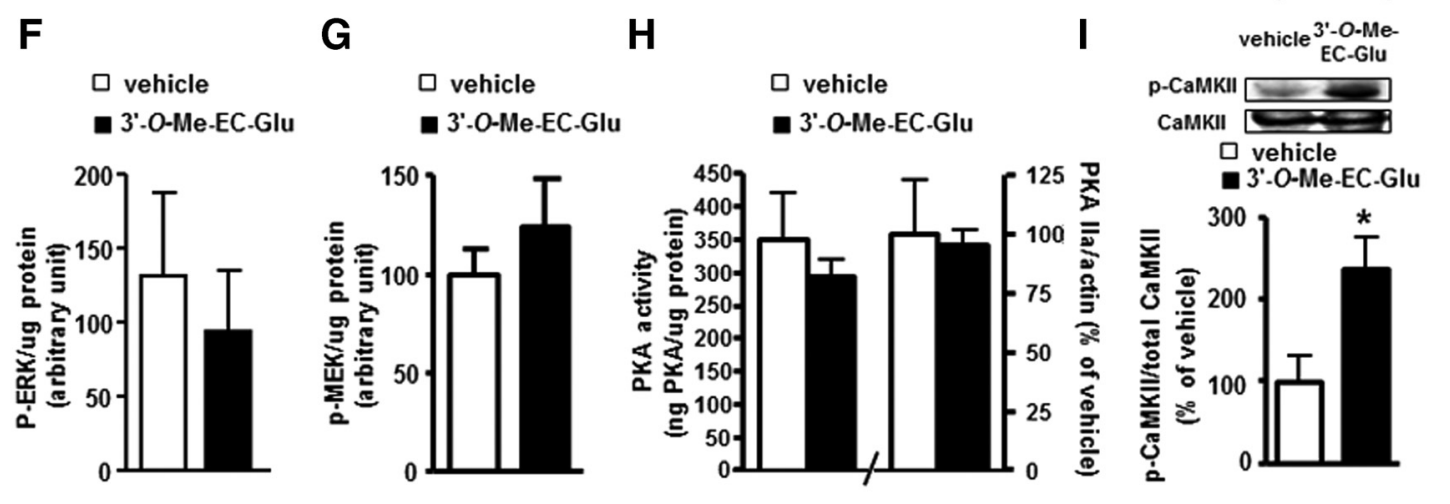

Figure 3. Biosynthetic 3'-0-Me-EC-5-0- $\beta$-glucuronide improves basal synaptic transmission and long-term potentiation coinciding with increased CREB hyperphosphorylation. $A-D$, The effect of $300 \mathrm{~nm} 3^{\prime}-0$-Me-EC-Gluc treatment on basal neuronal transmission and LTP in hippocampal slices from $A D(A, B)$ and wild-type $(W t)$ mice $(C, D)$. Arrow indicate the beginning of tetanus to induce LTP. E-I, The effect of 3'-0-Me-(-)-EC-5-0- $\beta$-glucuronide on CREB signaling pathway in hippocampal slices from old Tg2576 mice following $5 \mathrm{~h} 300 \mathrm{~nm} 33^{\prime}-0-\mathrm{Me}$-EC-Gluc treatment. The levels of phosphor-proteins of (REB phosphorylation at Ser133 (E), Erk1/2 phosphorylation at Thr185/Tyr187 (F), MEK phosphorylation at Ser222 (G). $\boldsymbol{H}, \boldsymbol{I}$, , PKA activity and protein content of PKA lla subunit expression $(\boldsymbol{H})$ and phosphor-CaMKII and total CaMKII $(\boldsymbol{I})$ in brain slices from Tg2576 mice. Inset, representative Western blot image of P-CaMKII and total CaMKII. * $p<0.05, n=5$ per group.

for methylated C and EC metabolites from Mo. Increases in AUC values for methylated EC glucuronides were also significantly $(p<0.05)$ higher from Mo $\left(216.0-952 \mathrm{nmol} / \mathrm{L}^{\star} \mathrm{h}\right)$ compared with GP $\left(187.7-241.7 \mathrm{nmol} / \mathrm{L}^{\star} \mathrm{h}\right)$. Similar increases were noted for methylated C glucuronides (Table 1).

Assessment of metabolite accumulation in rat brain tissue following $10 \mathrm{~d}$ of repeated dosing

A central issue in drug development for the CNS is whether the drug crosses the blood-brain barrier and whether sufficient concentrations of the drug reach the brain. We found trace contents (below levels for quantification) of free $\mathrm{C}$ and $\mathrm{EC}$, but much higher contents of $\mathrm{C}$ and EC phase II metabolites (both $O-\beta$ glucuronides and $O-\mathrm{Me}-\beta$-glucuronides) in the brain following repeated dosing with GP or Mo. The calculated total C and EC metabolite levels in the brain are 316.7 and $363.5 \mathrm{pmol} / \mathrm{g}$ following GP or Mo treatment, respectively (Fig. 2 B). Consistent with plasma PK data, we found much lower levels of total monomeric metabolites $(\sim 19.2 \mathrm{pmol} / \mathrm{g})$ in the brain following Po treatment (Fig. $2 B$ ). Figure $2 B$, inset, shows the MRM traces for C/EC-O- $\beta$-glucuronides $(465.1 \rightarrow 289.1 \mathrm{~m} / \mathrm{z})$ and $\mathrm{Me}-\mathrm{C} / \mathrm{EC}-\mathrm{O}-\beta$-glucuronides $(479.1 \rightarrow 303.1 \mathrm{~m} / \mathrm{z})$. Interestingly, higher concentrations of methylated $\mathrm{C}$ and EC compared with simple glucuronide derivatives were found in the brain following GP or Mo treatment, indicating either a preferential accumulation or metabolism of these methylated metabolites from the blood, or potential differences in the kinetics of transferring $\mathrm{C}$ and EC metabolite across the blood-brain barrier.
Biosynthetic 3'-O-Me-EC-Gluc improves basal synaptic transmission and LTP in hippocampal slices from AD mice We next explored whether PAC metabolites that accumulated in the brain might contribute to the benefit of Mo treatment in terms of improving cognitive function in Tg2576 AD mice. We selected 3'$\mathrm{O}$-Me-EC-Gluc for initial bioactivity studies based on evidence suggesting a potential role of $\mathrm{EC}$ in promoting cognitive function (van Praag et al., 2007) and that high contents of $3^{\prime}$-O-Me-EC-Gluc are found in the brain following long-term treatment with Mo (Fig. 2B). We explored whether 3'-O-Me-EC-Gluc might modulate LTP, which is one of the major cellular mechanisms known to play a key role in learning and memory functions (Bliss and Collingridge, 1993).

We biosynthetically generated 3'-O-Me-EC-Gluc by incubating commercially available $3^{\prime}$-O-Me-EC with recombinant human uridine diphosphate (UDP) glucuronosyltransferase 1 (UGT1A9) in the presence of UDP-glucuronic acid, and then HPLC purified the resultant 3'-O-Me-EC-Gluc (Fig. $2 C$ ). The authenticity of our biosynthetic $3^{\prime}-O-M e-E C-G l u c$ is validated by (1) its coelution with $3^{\prime}-\mathrm{O}-\mathrm{Me}-\mathrm{EC}-\mathrm{Gluc}$ found in vivo (Fig. $2 D$, left), (2) matching in-line MS-TOF spectra to in vivo metabolites (Fig. 2D, right), and (3) NMR analysis confirming the molecular structure.

We treated hippocampal slices from old Tg2576 mice that had demonstrated deficits both in basal synaptic transmission and LTP with $300 \mathrm{~nm}$ concentration of the biosynthetic 3'-O-MeEC-Gluc. We found 3'-O-Me-EC-Gluc treatment significantly increased basal synaptic transmission in the CA1 region of hippocampal slices compared with vehicle-treated control 
slices $(p<0.01$; Fig. $3 A$ ). Treatment with 3 '-O-Me-EC-Gluc resulted in a significantly increased LTP, expressed as a percentage of baseline fEPSP slope compared with the vehicle treatment $(225 \pm 15 \%$ vs $145 \pm 12 \%, p<0.01$; Fig. $3 B)$.

In parallel control studies using brain slices derived from agematched wild-type mice, we found that treatment with $3^{\prime}$-O-MeEC-Gluc did not have any effect on basal synaptic transmission or on LTP (Fig. 3C,D).

Our observations revealed, for the first time, that a braintargeted PAC metabolite is capable of restoring the strength of synaptic transmission and synaptic plasticity in the hippocampal formation, a brain region that is central to normal cognitive function as well as cognitive impairments in AD.

\section{$3^{\prime}$-O-Me-EC-Gluc improves LTP through cAMP response element binding protein signaling}

We continued to explore the molecular mechanism by which $3^{\prime}$-O-Me-EC-Gluc promotes LTP. Based on evidence that the cAMP response element binding protein (CREB) signaling pathway is critical for LTP and memory formation (Bartsch et al., 1998), we assessed the effect of $3^{\prime}$-O-Me-EC-Gluc on the regulation of the CREB signaling pathway. Using an ELISA-based assay, we quantified the level of phosphorylated [Ser133]-P-CREB as a direct reflection of CREB activation in the hippocampal formation. We found that treatment of hippocampal slices with $300 \mathrm{nM}$ 3'-O-Me-EC-Gluc significantly increased levels of [Ser133]phosphorylated active CREB levels compared with vehicle-treated control slices $(p<0.05$; Fig. $3 E)$. Increased phosphorylation and activation of CREB by 3'-O-Me-EC-Gluc was independently confirmed by Western blot analysis using an antibody specific to [Ser133]-P-CREB (data not shown).

Multiple pathways can lead to the phosphorylation and activation of CREB including extracellular signal-related protein kinase/ mitogen-activated protein (Erk/MAP) kinase pathway, protein kinase $\mathrm{A}$ (PKA) and $\mathrm{Ca}^{2+} /$ calmodulin-dependent protein kinases (CaMKs). Using a multiplex pathway signaling ELISA assay, we found that $3^{\prime}$-O-Me-EC-Gluc treatment did not modulate the phosphorylation status of Erk1/2 (Thr185/Tyr187) or MEK (Fig. 3F, G); nor did it affect PKA (both by PKA activity assay and PKA IIa protein content; Fig. $3 H$ ). However, examination of CaMKII by Western blot analysis showed that $3^{\prime}$-O-Me-EC-Gluc treatment significantly increased the level of [Thr286]-phosphorylated active CaMKII without influencing the level of total CaMKII (Fig. 3I), suggesting that 3'-O-Me-EC-Gluc-modulated CREB activation is, in part, mediated by the CaMKII signaling pathway.

\section{Discussion}

Age-related dementia, including AD, is one of the most persistent and devastating disabilities in the ever-aging population. In recent years, there has been an increasing interest in the potential value of polyphenolic compounds for preventing and/or treating AD. However, due to the complexity of these compounds and a limited understanding of their bioactivity, absorption, metabolism, and distribution to brain tissues, the development of effective polyphenolic compounds suitable for clinical application has been rather limited.

In this study, we showed that fractions of GP, namely Mo and Po, in vitro, interfere with the generation of soluble, neurotoxic $\mathrm{A} \beta$ oligomer species implicated in neuronal dysfunction in $\mathrm{AD}$ (Funke et al., 2007; Selkoe, 2008). However, in vivo studies revealed that only Mo is capable of improving spatial memory function and reducing $\mathrm{A} \beta$-mediated neuropathology in the brain following oral administration. This is mainly due to the fact that polyphenolic components and metabolites from Mo are bioavailable, while these components from Po are largely not bioavailable.

Pharmacokinetic studies illustrated that the primary circulating forms of polyphenols from Mo are C and EC monomeric glucuronides and methylated glucuronide metabolites. We demonstrated that repeated dosing of Mo resulted in the accumulation of $\mathrm{C}$ and $\mathrm{EC}$ metabolites in the brain with concentrations reaching $>300 \mathrm{pmol} / \mathrm{g}$. Moreover, a biosynthetic brain-targeted PAC metabolite, 3'-O-Me-EC-Gluc, at a physiologically relevant concentration, can significantly improve basal synaptic transmission and maintenance of LTP through mechanisms associated with activation of CREB signaling, a pathway involved in synaptic plasticity essential for learning and memory (Chrivia et al., 1993; Bartsch et al., 1998).

Our observation of C/EC-glucuronide metabolites in both plasma and perfused brain tissues in this study is consistent with observations by Abd El Mohsen and colleagues $(2002,2006)$ and Milbury and Kalt (2010), who have previously identified flavonoid glucuronides in perfused brain tissues of rodents and pigs. It is also well known that catechins can be metabolized to sulfate conjugates, which are often seen in urine and to a limited extent in blood and select tissues. However, to date, catechin sulfate metabolites have not been reported in brain tissues. In the present study, sulfate derivatives were not observed as major metabolites in the brain but it is possible that a small amount of sulfanated metabolites present and contribute to the benefits we observed in our study. Moreover, C/EC-glucuronide metabolites in the plasma might also have some beneficial impact on the brain through peripheral mechanisms, e.g., increases blood flow. These areas merit further investigation.

In conclusion, our studies suggest that chronic Mo treatment results in the accumulation of bioactive metabolites in the brain that are capable of reducing pathology and restoring neuronal function associated with learning and memory in the AD brain. Furthermore, our studies provide the first experimental evidence that a biosynthetic PAC metabolite can restore basal synaptic transmission and LTP. Future studies will clarify the identity of other specific bioactive PAC metabolites and their mechanisms of action, and will establish a scientific basis for the development of novel phytotherapeutics in the treatment of $\mathrm{AD}$ and other forms of dementia.

\section{Notes}

Supplemental material for this article is available at http://www. mountsinai.org/static_files/MSMC/Files/Faculty\%20Profile\%20Pdfs/ Wang\%20et\%20al\%20supplementary\%20figures.pdf. Supplemental data for in vitro PICUP assay, images of brain amyloid and NMR data for the epicatechin metabolite. This material has not been peer reviewed.

\section{References}

Abd El Mohsen MM, Kuhnle G, Rechner AR, Schroeter H, Rose S, Jenner P, Rice-Evans CA (2002) Uptake and metabolism of epicatechin and its access to the brain after oral ingestion. Free Radic Biol Med 33:1693-1702.

Abd El-Mohsen M, Bayele H, Kuhnle G, Gibson G, Debnam E, Kaila Srai S, Rice-Evans C, Spencer JP (2006) Distribution of [3H]trans-resveratrol in rat tissues following oral administration. Br J Nutr 96:62-70.

Bartsch D, Casadio A, Karl KA, Serodio P, Kandel ER (1998) CREB1 encodes a nuclear activator, a repressor, and a cytoplasmic modulator that form a regulatory unit critical for long-term facilitation. Cell 95:211-223.

Bliss TV, Collingridge GL (1993) A synaptic model of memory: long-term potentiation in the hippocampus. Nature 361:31-39.

Chapman PF, White GL, Jones MW, Cooper-Blacketer D, Marshall VJ, Irizarry M, Younkin L, Good MA, Bliss TV, Hyman BT, Younkin SG, 
Hsiao KK (1999) Impaired synaptic plasticity and learning in aged amyloid precursor protein transgenic mice. Nat Neurosci 2:271-276.

Chrivia JC, Kwok RP, Lamb N, Hagiwara M, Montminy MR, Goodman RH (1993) Phosphorylated CREB binds specifically to the nuclear protein CBP. Nature 365:855-859.

Feng WY (2006) Metabolism of green tea catechins: an overview. Curr Drug Metab 7:755-809.

Funke SA, Birkmann E, Henke F, Görtz P, Lange-Asschenfeldt C, Riesner D, Willbold D (2007) Single particle detection of Abeta aggregates associated with Alzheimer's disease. Biochem Biophys Res Commun 364:902-907.

Gong B, Vitolo OV, Trinchese F, Liu S, Shelanski M, Arancio O (2004) Persistent improvement in synaptic and cognitive functions in an $\mathrm{Alz}$ heimer mouse model after rolipram treatment. J Clin Invest 114:1624-1634.

Hartman RE, Shah A, Fagan AM, Schwetye KE, Parsadanian M, Schulman RN, Finn MB, Holtzman DM (2006) Pomegranate juice decreases amyloid load and improves behavior in a mouse model of Alzheimer's disease. Neurobiol Dis 24:506-515.

Milbury PE, Kalt W (2010) Xenobiotic metabolism and berry flavonoid transport across the blood-brain barrier. J Agric Food Chem 58:3950-3956.

Rezai-Zadeh K, Shytle D, Sun N, Mori T, Hou H, Jeanniton D, Ehrhart J, Townsend K, Zeng J, Morgan D, Hardy J, Town T, Tan J (2005) Green tea epigallocatechin-3-gallate (EGCG) modulates amyloid precursor protein cleavage and reduces cerebral amyloidosis in Alzheimer transgenic mice. J Neurosci 25:8807-8814.

Roura E, Andrés-Lacueva C, Jáuregui O, Badia E, Estruch R, IzquierdoPulido M, Lamuela-Raventós RM (2005) Rapid liquid chromatography tandem mass spectrometry assay to quantify plasma (-)-epicatechin metabolites after ingestion of a standard portion of cocoa beverage in humans. J Agric Food Chem 53:6190-6194.

Selkoe DJ (2008) Soluble oligomers of the amyloid beta-protein impair synaptic plasticity and behavior. Behav Brain Res 192:106-113.

Sharma V, Zhang C, Pasinetti G, Dixon R (2011) Fractionation of grape seed proanthocyanidins for bioactivity assessment. In: The biological activity of phytochemicals (Gang DR, ed), pp 33-46. NY: Springer.

Tsang C, Auger C, Mullen W, Bornet A, Rouanet JM, Crozier A, Teissedre PL (2005) The absorption, metabolism and excretion of flavan-3-ols and procyanidins following the ingestion of a grape seed extract by rats. $\mathrm{Br} \mathrm{J}$ Nutr 94:170-181.

van Praag H, Lucero MJ, Yeo GW, Stecker K, Heivand N, Zhao C, Yip E, Afanador M, Schroeter H, Hammerstone J, Gage FH (2007) Plantderived flavanol (-)epicatechin enhances angiogenesis and retention of spatial memory in mice. J Neurosci 27:5869-5878.

Wang J, Ho L, Qin W, Rocher AB, Seror I, Humala N, Maniar K, Dolios G, Wang R, Hof PR, Pasinetti GM (2005) Caloric restriction attenuates beta-amyloid neuropathology in a mouse model of Alzheimer's disease. FASEB J 19:659-661.

Wang J, Ho L, Chen L, Zhao Z, Zhao W, Qian X, Humala N, Seror I, Bartholomew S, Rosendorff C, Pasinetti GM (2007) Valsartan lowers brain beta-amyloid protein levels and improves spatial learning in a mouse model of Alzheimer disease. J Clin Invest 117:3393-3402.

Wang J, Ho L, Zhao W, Ono K, Rosensweig C, Chen L, Humala N, Teplow DB, Pasinetti GM (2008) Grape-derived polyphenolics prevent Abeta oligomerization and attenuate cognitive deterioration in a mouse model of Alzheimer’s disease. J Neurosci 28:6388-6392. 